\title{
Clima social, prosocialidad y violencia como predictores de inadaptación escolar en primaria
}

\author{
Cira Carrasco ${ }^{1}$ y $^{\mathrm{a}}$ Victoria Trianes ${ }^{2}$ \\ ${ }^{1}$ Colegio Infantil y Primaria "Doctor Fleming” Málaga \\ ${ }^{2}$ Universidad de Málaga (España)
}

\begin{abstract}
En este trabajo se pretende analizar la aportación que hacen la percepción del clima social, el comportamiento prosocial y la violencia escolar cotidiana sufrida y observada, informadas por parte del alumnado para predecir la adaptación socioemocional informada por profesores/as y padres o madres. Los participantes son 88 alumnos/as de 8-10 años de edad, de un colegio de la provincia de Málaga. Los resultados muestran que el alumnado evaluado presenta valores normalmente bajos en variables que indican inadaptación escolar y valores normativos en variables que se asocian a una buena adaptación escolar y social. Las correlaciones entre las variables predictoras y criterio muestran dos patrones diferentes: mientras que los datos de padres se asocian más activamente a las variables clima social y violencia sufrida en el caso de los profesores son prosocialidad y violencia sufrida. Los análisis de regresión demuestran también dos patrones diferentes para padres y para profesores, poniendo de manifiesto la importancia que tiene la violencia cotidiana sufrida en los resultados de adaptación o inadaptación escolar y social en alumnado que está en segundo ciclo de Educación Primaria, y la relevancia del comportamiento prosocial para predecir buenos resultados en el ámbito académico.
\end{abstract}

Palabras clave: Adaptación social, clima social, violencia escolar, comportamiento prosocial.

Social climate, pro-social behaviour and school violence as predictors of school inadaptation in Primary School. The general aim of this work is to analyse how students' perception of social climate, pro-social behaviour and the observation and experience of school violence contribute to predict their social and emotional adaptation reported by parents and teachers. The study has been conducted on a sample of 88 pupils between 8 to 10 years of age, belonging to a Primary School in the province of Málaga, Spain. The results show that the sample presents low marks in variables associated with poor social adaptation and standard marks in variables related to a successful social adaptation. The correlations between the predicting variables and criterion show two different patterns: meanwhile parents' data are more actively associated to social climate and the experience of violence, teachers' data are to pro-sociality and the experience of violence. The regression analysis also offers two different patterns for parents and teachers, stressing the influence of experienced daily school violence upon the results of social and educational adaptation and the importance of pro-social behaviour to predict good academic results of students in Primary School.

Key words: Social adaptation, social climate, school violence, pro-social behaviour.

Correspondencia: Cira Carrasco Romero. Colegio Infantil y Primaria "Doctor Fleming". C.P. 29016. Málaga (España). E-mail: ciracarrasco@ yahoo.es 
La inadaptación social y emocional supone riesgos importantes para un desarrollo armonioso y deseable en el contexto escolar. La inadaptación se asocia a sufrir prácticas de crianza no recomendables por parte de la familia (Dekovic, Janssens y Van As, 2003), a sufrir violencia experimentada como víctima (Perren y Alsaker, 2006), como agresor (Benítez y Justicia, 2006) u observador activo de la situación (Roeser y Eccles, 1998). Se asocia también a climas escolares conflictivos y a comportamientos insolidarios y egoístas (Slapak et al., 2000). Es por tanto un tipo de problema que debe evitarse en aras de conseguir una educación saludable para todo el alumnado, incluido los que presentan riesgos.

Hoy la psicología de la Educación se plantea examinar componentes de las experiencias que conducen a la inadaptación y a psicopatología en el alumnado. Conociendo las variables de riesgo, sobre todo las ubicadas en el contexto escolar, se podría intervenir con mayor eficacia para prevenir y tratar alumnado vulnerable al problema. Es el momento de plantearse enfoques complejos que incluyan diversas variables, a diversos niveles, que pueden sumar sus efectos para conseguir un resultado.

Entre las variables seleccionadas en este trabajo para estudiar su efecto en el problema de la inadaptación, está la violencia sufrida u observada. A lo largo de estos años el concepto de violencia escolar ha ido sufriendo cambios. En un comienzo, el concepto violencia se refería exclusivamente a violencia física, tras años de investigaciones el término se ha ampliado y perfilado, incluyendo también la violencia social o psicológica (Olweus, 1993). En un comienzo se estudió el comportamiento de acoso o abuso, mientras que ahora se estudia también la violencia escolar cotidiana. Se define ésta como comportamientos de baja intensidad pero de alta frecuencia que se producen diariamente en las relaciones recíprocas entre alumnos/as y también a veces, en las relaciones alumnos/as con profesores/as o viceversa (Trianes, 2004). La variable utilizada en el presente estudio es violencia cotidiana autoinformada sufrida y observada.

La violencia cotidiana en la escuela tiene consecuencias negativas para toda la Comunidad Educativa: agresores, víctimas y observadores y perjudica el clima social del centro (Defensor del Pueblo, 2007). Los docentes reconocen que los problemas de conducta afectan severamente las actividades docentes en el aula (Slapak et al., 2000).

La violencia afecta también a las relaciones de los diferentes miembros de la comunidad educativa impidiendo el adecuado clima social en el centro. Recientemente se ha diferenciado entre clima académico y clima social del centro escolar. En ese aspecto, el concepto de clima social es relativamente novedoso (Trianes, Blanca, de la Morena, Infante y Raya, 2006) y hace referencia, más allá de las relaciones mantenidas en temas académicos, a la calidad de las interacciones entre estudiantes-profesores/as y entre estudiantes-estudiantes (Emmons, Comer y Haynes, 1996) y a la percepción por parte de alumnos/as y profesores/as de bienestar personal, sentimientos positivos de sentirse aceptado y ser valioso para los demás en la convivencia diaria (Trianes, 2.000). 
Un clima positivo es necesario para poder impartir las materias académicas eficazmente. Las investigaciones plasman la relación inversa entre clima social y violencia escolar, y muestran como la mejora en el clima social es un medio eficaz para prevenir la violencia (Westling, 2002), asociándose a una disminución del riesgo de conductas agresivas y violentas que pudieran impactar negativamente en la vida del aula o del centro (Trianes et al., 2006) y presentando efectos positivos sobre el ajuste psicológico (Kuperminc, Leadbeater y Blatt, 2001) sobre todo en estudiantes que pueden tener riesgos de dificultades académicas, emocionales o comportamentales (Haynes, Emmons y Ben-Avie, 1997).

Otra variable que ha sido seleccionada en este trabajo por su relación, aunque negativa, con la violencia escolar es la conducta prosocial. Estudios llevados a cabo en niños/as entre 6 a 12 años muestran correlaciones negativas entre la conducta prosocial y las conductas verbales de rechazo (Pepler, Craig y Roberts, 1998). En clase, los profesores dan relevancia a la conducta prosocial ya que consideran buen alumnado al que comparte y colabora o coopera en las actividades de clase otros. La conducta prosocial ha sido objeto de estudio desde diversas perspectivas a lo largo de décadas. Aunque no existe consenso en la terminología - prosocialidad, conductas de ayuda, altruismo y cooperación, además de otros términos empleados- la mayoría de las investigaciones coinciden en referirse a las conductas prosociales como aquellas acciones voluntarias realizadas por las personas con el fin de ayudar y beneficiar a terceros (Eisenberg y Fabes, 1998). La conducta prosocial se caracteriza por implicar a veces algún costo, sacrificio o riesgo por parte de la persona que lo lleva a cabo (Roche, 1982) y el término incluye gran variedad de conductas como la generosidad, el altruismo, la compasión, proporcionar apoyo material o psicológico, compartir posesiones, participar en actividades para reducir las desigualdades e injusticias (Mussen y Eisenberg, 1997). El comportamiento de ayuda aumenta entre los 6 a 8 años pero disminuye de 9 a 12 años (Frydman y Ritucci, 1988). En cuanto a la influencia del género, las investigaciones concluyen que los niños/as tienden a ayudar más a los iguales del mismo género que a los del opuesto (Eisenberg y Mussen, 1989) aceptándose también que las niñas tienden a ser más prosociales, en términos generales, que los niños aunque no existe consenso al determinar el origen de estas diferencias (Eisenberg et al., 1998).

La conducta prosocial es un indicador de la adaptación escolar y social del alumnado (Guevara, Cabrera y Barrera, 2007). La adaptación social del alumnado tiene efectos positivos en el clima social del centro escolar mientras que la inadaptación influye negativamente (Barber, 2002), siendo pues una variable relevante en relación a la mejora de la convivencia escolar y el desarrollo integral del alumnado.

La aportación de este estudio es el análisis conjunto de tres importantes variables en el ámbito de explicación de la inadaptación o psicopatología infantil: la 
violencia diaria sufrida y observada en el colegio, la experiencia de clima social en el mismo, y el comportamiento prosocial, en positivo para explicar tentativamente la inadaptación o psicopatología en niños y niñas. Sería deseable poder contribuir a que, enfoques complejos actuales que busquen el origen de la inadaptación o la psicopatología infantil tengan en cuenta las variables estudiadas como relevantes en relación con la explicación pero también la prevención y el tratamiento de inadaptación o psicopatología infantil.

\section{Objetivos}

Este estudio pretende, en términos generales, analizar la aportación que hacen la percepción del clima social y de la violencia cotidiana sufrida y observada por parte del alumnado además del comportamiento prosocial para predecir la adaptación socioemocional informada por profesores y padres.

Por tanto, objetivos específicos de este trabajo son: a) describir los niveles que alcanzan en la muestra las variables de este estudio, que son clima social del centro, clima social de las relaciones con los profesores, violencia cotidiana sufrida, violencia cotidiana observada, y prosocialidad, y las variables de la inadaptación: problemas externalizados, problemas internalizados, problemas escolares, habilidades adaptativas y otros problemas; b) calcular las correlaciones entre las variables del clima social, de la violencia y del comportamiento prosocial (autoinformadas) y las de la prueba de inadaptación (informadas por padres y profesores); y c) El tercer objetivo de la investigación consiste en averiguar la capacidad de predicción que tienen las variables clima social, violencia y prosocialidad con respecto a la predicción de inadaptación. En concreto, se desarrollarán dos análisis de regresión, uno para obtener la predicción de la adaptación informada por padres o madres, y otro análisis para la predicción de la adaptación informada por profesores/as.

\section{Implicaciones de los objetivos}

En relación con el primer objetivo, se espera que la muestra estudiada alcance valores de normalidad al ser extraída de la población escolar normal sin ningún sesgo inicial de psicopatología o carácter específico.

En relación al segundo objetivo se espera que existan intercorrelaciones positivas y negativas entre las variables seleccionadas dependiendo su sentido de la naturaleza de dichas variables: relaciones positivas de la inadaptación con violencia y negativas con clima social y con comportamiento prosocial. En el caso de la escala de Habilidades adaptativas de la prueba de adaptación esperamos que demuestre correlaciones negativas con violencia y positivas con clima social y con comportamiento prosocial, de acuerdo a la experiencia pedagógica y a los resultados de estudios revisados que han servido de base para la presente investigación.

En relación al tercer objetivo y según las correlaciones encontradas previamente se espera que las variables clima social, comportamiento prosocial y 
violencia escolar cotidiana predigan con éxito la adaptación evaluada por profesores y con menos potencia la adaptación evaluada por padres, ya que esta se supone referida al contexto familiar.

\section{METODO}

\section{Participantes}

Este estudio se ha llevado a cabo en un colegio público de Málaga. Han participado un total de 88 alumnos/as de $2^{\circ}$ ciclo de Educación Primaria.: 51 niños (58\%) y 37 niñas (42\%); 66 españoles (75\%) y extranjeros (25\%), 39 de $3^{\circ} \mathrm{EP}(44.3 \%)$ y 49 de $4^{\circ}$ de EP $(55.7 \%)$ La edad está comprendida entre 7 y 10 años. También han participado activa y voluntariamente 4 profesores ( 2 hombres y 2 mujeres) y 88 familias.

\section{Instrumentos}

Con el fin de obtener información, lo más completa posible, sobre las variables de estudio, se han utilizado en esta investigación, diversas fuentes, asumiendo un enfoque multifuente en la investigación (Masten et al., 1995): progenitores, profesores, y el propio alumno han sido reconocidas a lo largo de diversas investigaciones como fuentes fiables y válidas para evaluar el comportamiento (Trianes, Blanca, García, Muñoz y Fernández, 2007). Los instrumentos aplicados han sido los siguientes:

Cuestionario del Clima Social del Centro Escolar, (Trianes et al., 2006). Tiene como objetivo el análisis del clima social del centro por parte del alumnado evaluando dos factores: 1) clima referente al centro, 8 ítems y 2) clima referente al profesorado, 6 ítems. Fuente: autoinforme.

Cuestionario de Violencia Escolar Cotidiana (Fernández, Trianes, de la Morena, Infante y Blanca, de la Universidad de Málaga (aceptado para publicación) que tienen por objetivo el análisis de la violencia escolar cotidiana por parte del alumnado evaluando dos factores: 1) la experiencia de sufrir violencia, 8 ítems y 2) la experiencia de observar violencia en el centro escolar, 5 ítems. Fuente: autoinforme.

PBQ. Cuestionario de Conducta Prosocial (Weir y Duvven, 1981) para la investigación sobre la conducta prosocial en niños de 5 a 11 años, 20 ítems. Fuente: autoinforme.

Cuestionario P-2. BASC, Sistema de evaluación de la conducta de niños/as de 6 a 11 años, adaptada al castellano por Santamaría (2004) para evaluar a) conductas adaptativas: adaptabilidad (8 ítems), liderazgo (10 ítems), habilidades sociales (14 ítems) y b) las conductas no adaptativas: agresividad (13 ítems), hiperactividad (9 ítems), problemas de conducta (10 ítems), ansiedad (7 ítems), depresión (12 ítems), somatización (14 ítems), atipicidad (12 ítems), retraimiento (9 ítems) y problemas de atención (7 ítems). Fuente: padres.

Cuestionario T-2. BASC, Sistema de evaluación de la conducta de niños/as de 6 a 11 años, adaptada al castellano por Santamaría (2004) para evaluar a) conductas adaptativas: adaptabilidad ( 7 ítems), liderazgo (9 ítems), habilidades sociales (12 ítems) 
y habilidades para el estudio (12 ítems) y b) las conductas no adaptativas: agresividad (14 ítems), hiperactividad (12 ítems), problemas de conducta (9 ítems), ansiedad (8 ítems), depresión (9 ítems), somatización (10 ítems), atipicidad (14 ítems), retraimiento ( 9 ítems), problemas de atención (9 ítems) y problemas de aprendizaje (9 ítems). Fuente: profesorado.

\section{Procedimiento}

Durante los cursos 2006-2008 se llevó a cabo la presente investigación solicitando y obteniendo los permisos pertinentes del claustro de profesores y del Consejo Escolar. Informadas las familias implicadas, se solicitó su participación, otorgando permiso por escrito y completando un cuestionario que se les entregó a los asistentes en la misma reunión. Las pruebas se administraron a todo el alumnado de $2^{\circ}$ ciclo de Educación Primaria, quienes participaron voluntariamente. El profesorado participante cumplimentó los cuestionarios tras una breve reunión.

\section{RESULTADOS}

Los resultados de estos análisis se exponen diferenciando las distintas fuentes empleadas, ya que, en función de las fuentes, se han utilizado unos instrumentos determinados. Así, puede verse en la tabla 1 los datos de las variables: clima social del centro, clima social en la relación con el profesorado, violencia cotidiana sufrida, violencia cotidiana observada y prosocialidad, todas ellas procedentes de autoinforme. Las variables de clima social presentan distribución normal y sus medias son altas respecto a la puntuación máxima posible. Las variables procedentes de la prueba sobre violencia escolar cotidiana, presentan también una distribución normal y sus medias están más centradas respecto a la puntuación máxima obtenida. En cuanto a la prosocialidad, presenta kurtosis hacia la derecha y su media es alta respecto a la máxima puntuación emitida.

Tabla 1. Estadísticos descriptivos de los factores informados por el alumnado

\begin{tabular}{lccccc}
\hline & Rango & Valor mín & Valor máx & $X$ & $D T$ \\
\hline Clima referente al centro & $8-40$ & 18 & 40 & 32.44 & 5.23 \\
Clima referente al profesorado & $6-30$ & 14 & 30 & 25.02 & 3.49 \\
Violencia sufrida & $8-40$ & 8 & 32 & 15.08 & 6.24 \\
Violencia observada & $5-25$ & 5 & 23 & 14.17 & 4.70 \\
Prosocialidad & $20-60$ & 30 & 60 & 50.95 & 6.17 \\
\hline
\end{tabular}

En el análisis descriptivo de las variables relativas a las conductas adaptativas y no adaptativas informadas por dos fuentes padres y profesorado (Tabla 2), dichas variables no se ajustan a la normalidad ya que describen síntomas psicopatológicos que no se distribuyen normalmente en la población general. Puede comentarse, en términos generales, que las medias de las variables de inadaptación no son altas siendo superiores 
las de las variables propias de adaptación y ajuste tanto de la de los padres como de la del profesorado.

Comparando en términos generales ambas fuentes puede comentarse que, si bien las puntuaciones medias son parecidas, en problemas interiorizados, los profesores puntúan más bajo que los padres justo lo contrario que ocurre en el caso de los problemas de atención y aprendizaje, en los que los padres puntúan más bajo que los profesores. Los padres puntúan más alto que los profesores en habilidades adaptativas, en las tres variables que comparten.

Tabla 2. Estadísticos descriptivos de la adaptación social informada por padres y profesorado

\begin{tabular}{|c|c|c|c|c|c|c|c|c|c|c|}
\hline & \multicolumn{5}{|c|}{ Padres } & \multicolumn{5}{|c|}{ Profesorado } \\
\hline & $\begin{array}{l}\stackrel{8}{80} \\
\tilde{\Xi} \\
\simeq\end{array}$ & 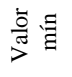 & 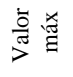 & $\rtimes$ & $\bar{\sigma}$ & $\begin{array}{l}0 \\
\stackrel{60}{\Xi} \\
\stackrel{\Xi}{\simeq}\end{array}$ & $\frac{\overline{0}}{\pi} \Xi$ & 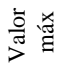 & $x$ & 5 \\
\hline - Agresividad & $13-52$ & 14 & 37 & 19.84 & 4.74 & $14-56$ & 14 & 50 & 20.58 & 8.87 \\
\hline - Hiperactividad & $9-36$ & 9 & 29 & 17.25 & 4.53 & $12-48$ & 12 & 46 & 20.17 & 8.41 \\
\hline - Problem conducta & $10-40$ & 10 & 31 & 14.00 & 3.62 & $9-36$ & 9 & 24 & 11.64 & 3.57 \\
\hline - Ansiedad & $7-28$ & 8 & 23 & 14.27 & 3.30 & $8-32$ & 8 & 27 & 12.17 & 3.77 \\
\hline - Depresión & $12-48$ & 12 & 30 & 17.00 & 3.42 & $9-36$ & 9 & 27 & 12.10 & 3.37 \\
\hline - Somatización & $14-56$ & 14 & 27 & 18.69 & 3.58 & $10-40$ & 10 & 23 & 11.08 & 2.08 \\
\hline - Problem atención & $7-28$ & 7 & 25 & 14.60 & 4.18 & $9-36$ & 9 & 36 & 19.86 & 7.85 \\
\hline - Probl aprendizaje & & & & & & $9-36$ & 9 & 36 & 18.14 & 7.94 \\
\hline - Atipicidad & $12-48$ & 12 & 27 & 16.85 & 3.10 & $14-56$ & 14 & 41 & 18.09 & 5.15 \\
\hline - Retraimiento & $9-36$ & 10 & 20 & 14.50 & 2.54 & $9-36$ & 9 & 28 & 14.21 & 4.93 \\
\hline - Adaptabilidad & $8-32$ & 17 & 32 & 26.53 & 3.44 & $7-28$ & 7 & 28 & 18.22 & 4.06 \\
\hline - Liderazgo & $10-40$ & 17 & 37 & 27.38 & 4.67 & $9-36$ & 9 & 32 & 19.94 & 6.36 \\
\hline - Habilid sociales & $14-56$ & 28 & 56 & 43.10 & 6.43 & $12-48$ & 13 & 47 & 29.57 & 9.37 \\
\hline - Habilid estudio & & & & & & $12-48$ & 14 & 44 & 29.45 & 8.66 \\
\hline
\end{tabular}

Con las puntuaciones obtenidas a partir de los instrumentos aplicados se calcularon los coeficientes de correlación de Pearson, con el fin de analizar las relaciones entre clima social, prosocialidad y violencia escolar cotidiana informada por el alumnado con la adaptación informada por los padres y el profesorado. Las relaciones existentes entre las variables predictoras informadas por el alumnado: clima social referente al centro, clima social referente al profesorado, violencia observada, violencia sufrida y prosocialidad y las variables dependientes tales como los factores de la conducta adaptativa y de la conducta no adaptativa difieren en función de la fuente de información padres y profesorado de forma evidente. Aunque en ambos agentes los factores del clima social y prosocialidad correlacionan de forma positiva con los factores componentes de la conducta adaptativa y de forma negativa con los factores componentes de la conducta inadaptativa; mientras que en el caso de los factores de la violencia escolar cotidiana se observa lo contrario.

Como puede verse en la tabla 3, la variable que más correlaciones presenta en los padres es la violencia sufrida. El análisis de correlación llevado a cabo arroja correlaciones positivas entre la violencia sufrida con las variables agresividad, hiperactividad, problemas de conducta, depresión, problemas de atención y atipicidad. 
En los datos informados por el profesorado (Tabla 4), la prosocialidad es el factor que presenta mayor número de correlaciones significativas. Puede observarse que la prosocialidad correlaciona significativamente de forma positiva con todos los factores de la conducta adaptativa: adaptabilidad, liderazgo, habilidades sociales y habilidades de estudio, mientras que correlaciona en negativo con agresividad, hiperactividad, problemas de conducta, problemas de atención, problemas de aprendizaje y atipicidad.

Tabla 3. Correlaciones de Pearson entre las variables predictoras en este trabajo y las correspondientes variables criterio informadas por los padres

\begin{tabular}{|c|c|c|c|c|c|}
\hline & $\begin{array}{l}\text { Clima } \\
\text { Centro }\end{array}$ & $\begin{array}{c}\text { Clima } \\
\text { Profesores }\end{array}$ & $\begin{array}{c}\text { Violencia } \\
\text { Sufrida } \\
\end{array}$ & $\begin{array}{c}\text { Violencia } \\
\text { Observada }\end{array}$ & Prosocialidad \\
\hline Agresividad & -.14 & -.14 & $.31 * *$ & $.25 *$ & $-.23^{*}$ \\
\hline Hiperactividad & -.09 & -.10 & $.30 * *$ & $.21^{\prime}$ & $-.29 * *$ \\
\hline Problema_conducta & -.11 & $-.24 *$ & $.40 * *$ & $.19^{\prime}$ & -.15 \\
\hline Ansiedad & -.06 & .07 & .14 & .15 & .01 \\
\hline Depresión & -.04 & -.12 & $.17^{\prime}$ & .13 & .01 \\
\hline Somatización & $.21 *$ & -.14 & .07 & -.08 & -.00 \\
\hline Problemas_atención & -.06 & $-.28 * *$ & $.32 * *$ & .13 & -.16 \\
\hline Atipicidad & .05 & -.14 & $.21 *$ & .08 & -.15 \\
\hline Retraimiento & .19 & .01 & .03 & -.02 & .09 \\
\hline Adaptabilidad & .03 & $.18^{\prime}$ & -.12 & $-.19^{\prime}$ & .01 \\
\hline Liderazgo & -.01 & $.23^{*}$ & .01 & -.19 & .16 \\
\hline Habilidades_sociales & .07 & $.29 * *$ & -.04 & .01 & $.25 *$ \\
\hline
\end{tabular}

Tabla 4. Correlaciones de Pearson entre las variables predictoras y las variables criterio informadas por el profesorado

\begin{tabular}{|c|c|c|c|c|c|}
\hline & $\begin{array}{l}\text { Clima } \\
\text { Centro }\end{array}$ & $\begin{array}{c}\text { Clima } \\
\text { Profesores }\end{array}$ & $\begin{array}{c}\text { Violencia } \\
\text { Sufrida }\end{array}$ & $\begin{array}{c}\text { Violencia } \\
\text { Observada }\end{array}$ & Prosocialidad \\
\hline Agresividad & -.02 & -.06 & $.35^{* * *}$ & .14 & $-.20^{\prime}$ \\
\hline Hiperactividad & -.00 & -.18, & $.29 * *$ & .00 & $-.33 * *$ \\
\hline Problema_conducta & -.00 & -.11 & $.35 * *$ & .11 & $-.25 *$ \\
\hline Ansiedad & .04 & .11 & -.15 & -.17 & .01 \\
\hline Depresión & .01 & -.04 & $.21^{\prime}$ & -.01 & -.14 \\
\hline Somatización & -.09 & -.15 & -.09 & $-.28 * *$ & -.15 \\
\hline Problemas_atención & -.03 & -.14 & $.29 * *$ & .04 & $-.28 * *$ \\
\hline Proble_aprendizaje & -.03 & -.14 & .15 & -.11 & $-.29 * *$ \\
\hline Atipicidad & .03 & -.13 & -.01 & -.20 & $-.28 * *$ \\
\hline Retraimiento & -.02 & .01 & -.05 & -.17 & -.15 \\
\hline Adaptabilidad & -.01 & .10 & $-.39 * *$ & -.11 & $.34 * *$ \\
\hline Liderazgo & -.08 & .07 & $-.36 * *$ & -.18 & $.37 * *$ \\
\hline Habilidades_sociales & -.09 & .05 & $-.41 * *$ & $-.27 *$ & $.30 * *$ \\
\hline Habilidades estudio & .09 & $.32 * *$ & $-.29 * *$ & .02 & $.41 * *$ \\
\hline
\end{tabular}

Por último, se procedió al análisis de regresión sobre la adaptación a partir de las puntuaciones de clima social, prosocialidad y violencia cotidiana escolar informada por el alumnado. Se han desarrollado dos análisis siguiendo el método de pasos sucesivos: uno para obtener la predicción de la adaptación informada por padres, y otro análisis para la predicción de la adaptación informada por profesores.

Primero se efectuó el análisis de regresión para obtener la predicción de la adaptación informada por padres. Violencia sufrida y clima de profesores son las variables que más intervienen en predicciones de variables dependientes tanto en 
solitario como interviniendo juntas. A continuación se exponen las puntuaciones $R^{2}$ y las ecuaciones de regresión obtenidas a partir del modelo expuesto informado por padres/madres.

Tabla 5. Variables predichas con su $\mathrm{R}^{2}$ y ecuación de regresión. Fuente: padres

\begin{tabular}{lll}
\hline \multicolumn{1}{c}{ Variable predicha } & $R^{2}$ & Ecuaciones de regresión \\
\hline Agresividad & .09 & $\mathrm{y}=16.30+.30$ violencia sufrida \\
Hiperactividad & .15 & $\mathrm{y}=24.13+.27$ violencia sufrida -.26 prosocialidad \\
Probl conducta & .22 & $\mathrm{y}=16.96+.40$ violencia sufrida -.25 clima profesores \\
Somatización & .06 & $\mathrm{y}=18.15+.27$ clima centro -.21 clima profesores \\
Probl atención & .18 & $\mathrm{y}=19.79+.32$ violencia sufrida -.28 clima profesores \\
Atipicidad & .04 & $\mathrm{y}=15.27+.21$ violencia sufrida \\
Liderazgo & .05 & $\mathrm{y}=19.77+.27$ clima profesores \\
Habilidades sociales & .08 & $\mathrm{y}=29.77+.28$ clima profesores \\
\hline
\end{tabular}

Finalmente se efectuó el análisis de regresión para obtener la predicción de la adaptación informada por el profesorado. Violencia sufrida junto con Prosocialidad son las variables predictoras que más intervienen en predicciones tanto en solitario como interviniendo juntas. En la tabla 6 se exponen las puntuaciones $R^{2}$ y las ecuaciones de regresión obtenidas a partir del modelo expuesto informado por el profesorado.

Tabla 6. Variables predichas con su $R^{2}$ y ecuación de regresión. Fuente profesorado

\begin{tabular}{lll}
\hline \multicolumn{1}{c}{ Variable predicha } & $R^{2}$ & \multicolumn{1}{c}{ Ecuaciones de regresión } \\
\hline Agresividad & .12 & $\mathrm{y}=13.07+.35$ violencia sufrida \\
Hiperactividad & .17 & $\mathrm{y}=36.19+.25$ violencia sufrida -.30 prosocialidad \\
Probl conducta & .16 & $\mathrm{y}=15.38+.32$ violencia sufrida -.22 prosocialidad \\
Somatización & .07 & $\mathrm{y}=12.83-.28$ violencia observada \\
Probl atención & .15 & $\mathrm{y}=31.60+.26$ violencia sufrida -.25 prosocialidad \\
Probl aprendizaje & .08 & $\mathrm{y}=36.92-.28$ prosocialidad \\
Atipicidad & .07 & $\mathrm{y}=29.91-.27$ prosocialidad \\
Adaptabilidad & .24 & $\mathrm{y}=11.45-.35$ violencia sufrida +.30 prosocialidad \\
Liderazgo & .23 & $\mathrm{y}=7.28-.32$ violencia sufrida +.33 prosocialidad \\
Habilidades sociales & .23 & $\mathrm{y}=18.33-.38$ violencia sufrida +.25 prosocialidad \\
Habilidades estudio & .27 & $\mathrm{y}=-1.35-.26$ violencia sufrida +.31 prosocialidad +.22 clima profesorado \\
\hline
\end{tabular}

\section{DISCUSION}

Para mayor claridad se va a proceder a dividir la discusión según los objetivos propuestos. En primer lugar, se describirán los niveles que alcanzan en la muestra las distintas variables. Dado que la muestra procede de un centro escolar cuyo contexto sociocultural y económico se ubica en clase media, con bienestar económico, el alumnado evaluado presenta valores normalmente bajos en variables que indican inadaptación escolar y normalmente más altos en variables que se asocian a una buena adaptación escolar y social.

En cuanto al objetivo segundo, calcular las correlaciones entre las variables independientes del clima social, de la violencia y del comportamiento prosocial (autoinformadas) y las dependientes de la prueba de inadaptación (informadas por padres 
y profesores); éstas han mostrado, en términos generales, diferentes patrones en los datos de padres y de profesorado.

Cabe comentar y con prudencia pues son correlaciones simples, que en los padres la variable del clima social escolar desempeña un papel más activo, produciendo mayor número de correlaciones significativas ( 6 frente a 2 ) posiblemente debido a que padres y profesores utilizan diferentes criterios para la valoración del comportamiento de los niños (Veenstra et al., 2008).

También difieren los datos, a favor de un mayor número de correlaciones en los profesores que en los padres, en las variables violencia sufrida (9 frente a 6) y prosocialidad (10 frente a 3). Respecto a violencia sufrida este resultado alude a que los profesores son más conscientes de que existen alumnos que sufren o ejercen violencia diaria. Respecto a la prosocialidad, los profesores valoran más este comportamiento ya que forma parte de los asociados a la adaptación escolar (Trianes, de la Morena y Muñoz, 1999).

Ambas fuentes valoran la relación entre la prosocialidad y las habilidades sociales, indicando como las habilidades sociales son apreciadas tanto en casa como en el colegio. Algunos estudios teóricos recomiendan trabajar el desarrollo de la habilidades sociales como medio para fomentar la adaptación y prevenir los conflictos entre iguales (López de Dicastillo, Iriarte y González, 2007). Además, el comportamiento prosocial es visto formando parte del constructo competencia social en algunas tipologías de habilidades sociales (Merrell y Gimpel, 1998).

Comentando en términos más específicos, existen correlaciones positivas, en los datos de los padres, entre el clima social relativo a las buenas relaciones con el profesorado y variables propias de la adaptación. Los resultados de otros estudios también muestran que un ambiente positivo se relaciona con un incremento en conductas amistosas y cooperación (Morrinson, Furlong y Morrinson, 1994). El clima con el profesorado se relaciona en positivo con adaptabilidad, liderazgo y habilidades sociales evaluadas por padres y con habilidades de estudio evaluadas por el profesorado. Otros investigadores encuentran también que unas relaciones positivas con el profesorado son un factor relevante para la adaptación escolar y la salud psicológica de los escolares (Trianes et al., 2006).

Continuando con los datos de padres versus profesorado, se exhiben correlaciones similares de los padres con respecto a los profesores en las relaciones de las variables agresividad, hiperactividad, problemas de conducta, depresión y problemas de atención, con respecto al autoinforme de violencia sufrida. Esto indica la relevancia de los problemas de adaptación escolar evaluados, ya que esta es reconocida tanto por ambas fuentes. Otras investigaciones encuentran también que algunos problemas de adaptación escolar se asocian a sufrir violencia por parte de los iguales (Beale, 2001). Cabe además señalar que mientras los padres han producido correlaciones de la violencia 
sufrida con variables de inadaptación, los profesores producen además correlaciones que tienen en cuenta variables positivas.

En los datos de los padres, la variable de violencia observada correlaciona con agresividad, hiperactividad, problemas de conducta y en negativo con adaptabilidad mientras que en el caso del profesorado, solo correlaciona en negativo con somatización, liderazgo y con habilidades sociales. Comparada con la violencia sufrida, la violencia observada produce menos correlaciones significativas, quizás es evaluada como algo normal por los alumnos en general, excepto por los agresivos y los más hábiles, indicando una habituación (O’Keefe, 1997) hacia la violencia observada de modo que las puntuaciones no son altas.

Finalmente, en relación a la prosocialidad, los profesores producen más correlaciones tanto con variables negativas como con habilidades positivas. Esto puede atribuirse a que la fuente profesor es quizás más sensible al comportamiento prosocial, ya que su desarrollo está presente en objetivos escolares de primaria y representa un comportamiento muy valorado en la escuela. En otras investigaciones la prosocialidad correlaciona positivamente con habilidades de estudios y relaciones sociales (Veenstra et al., 2008). Cabe resaltar que son las habilidades sociales la única variable que desempeña el papel de una correlación positiva con prosocialidad tanto en padres como en profesores, enfatizando la importancia de las habilidades sociales ya que promueven actitudes y conductas cooperativas, altruistas y democráticas (Trianes y FernándezFigarés, 2001). Por último, prosocialidad correlaciona en negativo con hiperactividad y agresividad en ambas fuentes y con problemas de conducta en el profesorado. Posiblemente ese síndrome produce muchos problemas en la interacción diaria en el centro y tratándose de alumnado aún pequeño no hay lugar para comportamientos de ayuda cuando esos niños están luchando por desarrollar un mayor control de su conducta y de su mente.

En cuanto al tercer objetivo relativo a los análisis de predicción sobre las variables criterio, se obtienen dos patrones diferentes, el de las puntuaciones de los padres y el del profesorado.

La variable violencia sufrida tiene un papel relevante, predice variables de inadaptación, prácticamente todas las que entran en dicho análisis, tanto en los datos de padres como en los profesores y, en negativo, todas las variables de adaptación en los datos del profesorado señalando el efecto demoledor del hecho de sufrir violencia cotidiana en la escuela (Trianes, 2004): disminución de autoestima, estados de ansiedad e incluso cuadros depresivos lo que dificulta la integración en el medio escolar y disminuye el del rendimiento académico (Perren y Alsaker, 2006).

El clima social referente al profesorado juega un papel relevante en los datos de padres, la cual predice en solitario prácticamente todas las variables de adaptación que entran en el análisis y, en negativo, algunas de las variables de inadaptación. El 
clima positivo referente al profesorado se asocia a menor puntuación en problemas de conducta y problemas de atención (Kuperminc, Leadbeater, Emmons y Blatt, 1997) y una mayor adaptación escolar (Haynes et al., 1997).

En cuanto al papel también relevante que desempeña la prosocialidad, en los datos del profesorado, ya se ha discutido que puede ser que el profesor valore más este tipo de comportamiento ya que está presente con frecuencia en el contexto escolar, como criterio. La prosocialidad también predice en negativo, es decir, los niños poco prosociales tienen más riesgo de ser valorados en negativo por profesorado (Longobardi, Pasta y Sclavo, 2008) y padres. El profesorado asocia el comportamiento prosocial o el antisocial a la realización de las tareas académicas y a las relaciones entre iguales (Veenstra et al., 2008).

Como conclusiones de este trabajo se pone de manifiesto la importancia que tiene la violencia cotidiana sufrida en los resultados de adaptación o inadaptación escolar y social, en alumnado que está en segundo ciclo de Educación Primaria. El comportamiento prosocial juega un importante papel para predecir buenos resultados en el ámbito académico. Desde el punto de vista de la intervención psicoeducativa este resultado apunta hacia la importancia de enseñar habilidades sociales, de comportamiento prosocial, y de estudio para prevenir, en alumnado de riesgo, el sufrir violencia y fastidios diarios por parte de los/as compañeros.

Es importante señalar que el alumnado participante en esta investigación ha sido más pequeño en edad que los participantes habituales en este tipo de trabajo. Han sido capaces de contestar con sinceridad, revelando el hecho incuestionable de que esa violencia cotidiana existe en estos niveles educativos tempranos. Hay que añadir que este trabajo se ha hecho en un centro escolar no problemático. Quizás no hay acosos ni comportamientos de violencia grave, pero esta violencia cotidiana está presente a menos que exista una intervención decidida del profesorado para producir aulas sin violencia

Para señalar limitaciones de esta investigación, en la próxima, tesis doctoral de la autora de este trabajo, habrá que tener en cuenta el utilizar instrumentos más breves para que el profesorado y padres los contesten. Finalmente señalar que el vocabulario de las pruebas de clima social y de violencia cotidiana era algo elevado para las edades de las muestras.

En cuanto a líneas futuras, es intención de la autora validar y proponer la utilización de un programa educativo que converja con los principios orientadores del Plan Andaluz de Cultura de Paz y Noviolencia: "primero, promover la paz como acción colectiva e individual, segundo, saber convivir con los conflictos y proponer soluciones creativas y pacíficas a los mismos; y tercero, detener, disminuir y prevenir las manifestaciones de la violencia". 


\section{REFERENCIAS}

Beale, A.V. (2001). 'BullyBusters': Using drama to empower students to take a stand against bullying behavior. Professional School Counseling, 4, 300-305.

Barber, B.K. (2002). Intrusive Parenting. En How psychological control affects children and adolescents ( $\mathrm{pp}$ 15-22). Washington: American Psychological Association.

Defensor del Pueblo (2007). Informes, estudios y documentos. Violencia escolar: el maltrato entre iguales en la Educación Secundaria Obligatoria 1999-2006. Madrid: Defensor del Pueblo.

Dekovic, M., Janssens, J. y Van As, N. (2003). Family Predictors of Antisocial Behavior in Adolescence. Family Process, 42, 223-235.

Eisenberg, N. y Fabes, R.A. (1998). Prosocial development. En W. Damon y N. Eisenberg (Eds.), Handbook of child psychology: Social, emotional, and personality development (pp. 701-778) (5th ed.). New York: Wiley.

Eisenberg, N. y Mussen, P. (1989). The roots of prosocial behavior in children. Cambridge: Cambridge University Press.

Emmons, C.L., Comer, J.P. y Haynes, N.M. (1996). Translating theory into practice: Comer's theory of school reform. En J.P. Comer, N.M. Haynes, E. Joyner y M. Ben-Avie (Eds.), Rallying the whole village (pp. 27-41). Nueva York: Teachers College Press.

Fernández, F.J., Trianes, M.V., de la Morena, L., Escobar, M., Infante, L. y Blanca, M.J. Un nuevo instrumento de evaluación de violencia escolar cotidiana (CUVECO). Aceptado para publicarse en Anales de Psicología.

Frydman, M. y Ritucci, G. (1988). Le dévelopment de l'attitude: experimentation et evaluation d' un programme centre sur le comportement d'aide. Enfance, 41(3-4), 73-85.

Guevara, I.P., Cabrera, V.E. y Barrera, F. (2007). Contextual factors and moral emotions as predictors of psychological adjusment in the adolescence. Universitas Psychologica, 6(2), 269-283.

Haynes, N.M., Emmons, C.L. y Ben-Avie, M. (1997). School climate as a factor in student adjustment and achievement. Journal of Educational and Psychological Consultation, 8, 321-329.

Kuperminc, G.P., Leadbeater, B.J. y Blatt, S.J. (2001). School social climate and individual differences in vulnerability to psychopathology among middle school students. Journal of School Psychology, 39 (2), 141-159.

Kuperminc, G.P., Leadbeater, B.J., Emmons, C.L. y Blatt, S.J. (1997). Perceived school climate and difficulties in the social adjustment of middle school students. Applied Developmental Science, 1, 76-88.

Longobardi, C., Pasta, T. y Sclavo, E. (2008). The educative relationship in primary school. European Journal of Education and Psychology, 1(2), 5-18.

López de Dicastillo, N., Iriarte, C. y González, M.C. (2007). El desarrollo de la Competencia Social como vía de prevención de los conflictos en el aula. Típica 3(1), 1-11.

Masten, A.S., Coatsworth, J.D., Neeman, J., Gest, S.D., Tellegen, A. y Garmezy, N. (1995). The structure and coherence of competence from children through adolescence. Child Development, 66, 1635-1659.

Merrell, K.W. y Gimpel, G.A. (1998). Social skills of children and adolescents: Conceptualization, assessment, treatment. Mahwah, NJ: Lawrence Erlbaum Associates.

Morrison, G., Furlong, M.J. y Morrison, R.L. (1994). School violence to school safety: reframing the issue for school psychologists. School Psychology Review, 23(2), 236-256.

Musen, P. y Eisenberb, N. (1997). Roots of caring, sharing and helping: The development of prosocial behavior in children. San Francisco: Freeman. 
O'Keefe, M. (1997). Adolescents' exposure to community and school violence: Prevalence and behavioral correlates. Journal of Adolescent Health, 20, 368-376.

Olweus, D. (1993). Bullying at school: What we know and what we can do. Oxford: Blackwell.

Pepler, D.J., Craig, W. y Roberts, W. (1998). Observations of aggressive and nonaggressive children on the playground. Merril Palmer Quarterly, 44(1), 55-76

Perren, S. y Alsaker, F.D. (2006). Social behavior and peer relationship of victims, bully victims, and bullies in kindergarten. Journal of Child Psychology and Psychiartry, 47(1), 45-57.

Roche, R. (1982). Los orígenes de la conducta altruista en niños. Aspectos educativos y televisión en familia. Infancia y Aprendizaje, 19-20, 101-114.

Roeser, R.W. y Eccles, J.S. (1998). Adolescents' perceptions of middle school: Relation to longitudinal changes in academic and psychological adjustment. Journal of Research on Adolescence, 8, 123-158.

Slapak, S., Sautu, R., Cervone, N., Luzzi, A.M., Moraleja, N., Padawer, M., Ramos, L., Rodríguez Nuñez, M.V., D’Onofrio, M.G. y Martínez-Mendoza, R. (2000). El papel de la educación en la socialización de niños con problemas de conducta. Psico-logos, 10, 517.

Trianes, M.V. (2000). Violencia en contextos escolares. Málaga: Aljibe.

Trianes, M.V. (2004). Contextos de la violencia juvenil en España. Ponencia presentada en la VIII Reunión Internacional sobre Biología y Sociología de la violencia: Violencia y Juventud. Centro Reina Sofía para el Estudio de la Violencia, Valencia.

Trianes, M.V., Blanca, M.J., de la Morena, L., Infante, L. y Raya, S. (2006). Un cuestionario para evaluar el clima social del centro escolar, Psicothema, 18(2), 272-277.

Trianes, M.V., Blanca, M.J., García, B., Muñoz, A. y Fernández, F.J. (2007). Análisis del comportamiento asertivo con adultos: un modelo de relaciones entre componentes y fuentes. Infancia y Aprendizaje, 30(2), 163-182.

Trianes, M.V., de la Morena, M. L. y Muñoz, A. (1999). Inadaptación social. En Relaciones sociales y prevención de la inadaptación social y escolar (pp. 45-72). Aljibe: Málaga.

Trianes, M.V. y Fernández-Figarés, C. (2001). Aprender a ser personas y a convivir. Un programa para secundaria. Bilbao: Desclée de Brouwer.

Veenstra, R., Lindenberg, S., Oldehinkel, A., De Winter, A., Verhulst, F. y Ormel, J. (2008). Prosocial and Antisocial Behavior in Preadolescence: Teachers' and Parents' Perceptions of the Behavior of Girls and Boys. International Journal of Behavioral Development, 32(3), 243-251.

Westling, M. (2002). A two level analisis of classroom climate in relation to social context, group composition and organization of special support. Learning Environments Research, 5, 253-274.

Recibido: 2 de enero de 2010

Recepción Modificaciones: 15 de marzo de 2010

Aceptado: 20 de marzo de 2010 